\title{
Membrane transporters studied by EPR spectroscopy: structure determination and elucidation of functional dynamics
}

\author{
Anna Mullen*, Jenny Hall*, Janika Diegel*, Isa Hassan*, Adam Fey* and Fraser \\ MacMillan*1 \\ *Henry Wellcome Unit for Biological EPR, School of Chemistry, University of East Anglia, \\ Norwich, U.K.
}

\begin{abstract}
During their mechanistic cycles membrane transporters often undergo extensive conformational changes, sampling a range of orientations, in order to complete their function. Such membrane transporters present somewhat of a challenge to conventional structural studies; indeed, crystallization of membrane-associated proteins sometimes require conditions that vary vastly from their native environments. Moreover, this technique currently only allows for visualization of single selected conformations during any one experiment. EPR spectroscopy is a magnetic resonance technique that offers a unique opportunity to study structural, environmental and dynamic properties of such proteins in their native membrane environments, as well as readily sampling their substrate-binding-induced dynamic conformational changes especially through complementary computational analyses. Here we present a review of recent studies that utilize a variety of EPR techniques in order to investigate both the structure and dynamics of a range of membrane transporters and associated proteins, focusing on both primary (ABC-type transporters) and secondary active transporters which were key interest areas of the late Professor Stephen Baldwin to whom this review is dedicated.
\end{abstract}

\section{Introduction}

Membrane transporter proteins comprise a wide variety of proteins which underpin a diverse range of functions - from the import of vital solutes to the efflux of a multitude of drugs - making this class of proteins a clear target for a plethora of therapeutic treatments in the bid to tackle a myriad of medical challenges. Resolving their 3D structures at a molecular level is an integral part of understanding their functional mechanisms. However, the issues that arise when attempting to solve the structures of such dynamic proteins often render the more typical methods unsuccessful. Such problems include the fact that it can be difficult to solubilize membrane-associated proteins for crystallization, and that X-ray crystallography relies on the arrangement of protein into a regular assembly. This often means that only single conformations can be sampled, leaving much of the dynamic cycle of these proteins unobserved and continually debated [1]. Additionally, membrane transporters may consist of large proteins, or can exist as multidomain complexes making them difficult to measure for size-limited methods such as NMR spectroscopy (though recent advances in solid state NMR are starting to minimize this limitation) [2]. There are numerous examples in the literature and in the PDB database, which demonstrate that membrane proteins are also especially susceptible to conformational changes due to environmental changes e.g. when being extracted from their native membrane [3].

There are only a few biophysical methods which can provide both structural and dynamic information on the molecular architectures, offering reliable and precise distance determination in the range 1.5-10 nm. EPR spectroscopy is one such technique (another being the fluorescencebased technique FRET spectroscopy). Both are now being more widely used, especially in combination with molecular dynamics approaches as complementary structural biology techniques, 
being called upon to overcome such obstacles mentioned above. The readers are referred to an excellent recent review of the application of single molecule FRET techniques to study $A B C$ transporters [4]. Here we will only focus on EPR, which can be harnessed in many ways to also provide structural constraints that aid visualization of the structure and dynamics of a protein in a range of states throughout its mechanistic cycle, as will be demonstrated in this review. Importantly, the method can also be applied to instances where the protein is situated within a membrane environment (either in vivo or reconstituted into proteoliposomes or even styrene maleic acid lipid particles, SMALPs [5]), allowing it to be used to obtain information about the protein in or as close to its native crowded environment as possible. The studies reviewed here aim to demonstrate the application of EPR to investigate the structural elements and mechanistic dynamics of a range of primary ( $A B C$-type transporters) and secondary active membrane transporters.

EPR spectroscopy is a spectroscopic technique that detects the interaction of unpaired electrons with microwave radiation within a magnetic field. Initially developed as a physical magnetic resonance spectroscopy following its discovery in the USSR in 1944, it soon developed in a key biophysical method for studying biological systems contain- ing intrinsic paramagnetic species, especially those involved in bioenergetic electron transfer and metalloproteins. The development of this method over the past seven decades has resulted in the emergence of a broad range of techniques that can be employed to study paramagnetic species and their surrounding environments [e.g. 6-8]. These methods have been reviewed in several excellent monographs and review articles over the past 10-20 years and the reader is referred to the following review articles and references therein [9-12].

Arguably one of the major turning points in the development of EPR methodology from being a rather niche technique into a key structural biology technique was the introduction of site-directed spin labelling (SDSL). This is a technique that allows attachment of a stable paramagnetic probe to a specific site (often made possible using site-directed mutagenesis) within proteins or nucleic acids without any intrinsic paramagnetic centres, thus making previously EPR-inactive diamagnetic proteins now accessible to this technique. One of the leading figures in this development is Prof. Wayne Hubbell (UCLA, USA) who has reviewed recent developments in the field [13]. Much of these recent advances have resulted from essential improvements in instrumentation, especially in sensitivity $[14,15]$. Compared with NMR or X-ray studies the SDSL methodology does not yet [but see 14-16] provide global structural models but rather contributes sparse structural constraints which require essential supporting analysis packages [e.g. 17,18]. Clearly questions can be asked with regard to the site-directed modification of proteins:- does a labelled protein still function correctly; how physiologically relevant is measuring a distance (or distances) at cryogenic temperatures; are purified, detergent-solubilized membrane proteins true reflections of a functional protein in a membrane, however with that in mind here we aim to focus on the key information that has been extracted from the application of EPR spectroscopy to the study of a range of primary (ABC-type transporters) and secondary active membrane transporters especially determination of longer distances [19], distance editing [20], resolving not only distances but also relative orientations [2123], measuring distances under physiological conditions [24] or in native environments (whole cells or oocytes) $[25,26]$. To summarize, EPR and especially in combination with SDSL is able to provide a wealth of detailed information both for the mechanistic structural biologist and the computational chemist to contribute to advancing our understanding of membrane transporters (see Figure 1).

\section{Membrane transport proteins}

We will not aim to cover all the published EPR data on the large family of membrane transporters since this is a vast area. Instead we aim to focus on recent developments on a few key areas 
including primary active transport and ATP-binding cassettes (ABC) as well as facilitated diffusion/secondary active transport systems. Figure 2 shows some examples of the families of structures of the various transporters for which we are reporting spectroscopic data.

\section{Primary active transport and ATP-binding cassettes}

\section{Substrate-binding proteins}

Also known as periplasmic-binding proteins (PBPs), substrate-binding proteins (SBPs) are a class of proteins that form complexes with membrane proteins. Commonly used for transport or signal transduction, SBPs can be found to be associated with ATP-binding cassette (ABC) transporters [27] and more recently in other membrane protein complexes $[28,29]$.

EPR studies of SBPs have been mainly limited to the maltose transporter MalEFGK2. The maltose-binding protein (MBP) is associated with the ATP-binding MalFGK2. Hall et al. [30] used both room temperature (RT) continuous wave (cw)-EPR and low temperature EPR techniques to demonstrate that there are two modes of ligand binding in the MBP; one being active and the other inactive, depending on the ligand orientation. They argue that the inactive mode hinders the closure of MalE, which prevents the complex from interacting with the inner membrane domain. They found that ligand binding did not affect the mobility of the spin labels attached to the protein, and deduced that the spectral broadening which occurred upon the addition of maltose was a result of an increase in spin-spin interaction in the double spin-labelled proteins. Using low temperature EPR techniques they found that upon maltose binding the

spin distance between the two domains changed from 16.5 to $10.5 \mathrm{~A}^{\circ}\left(1 \mathrm{~A}^{\circ}=0.1 \mathrm{~nm}\right)$.

Further to this, Austermuhle et al. [31] used SDSL techniques to study the interaction of MBP with the transmembrane domain of the transporter. ATP hydrolysis during the closure of the MalK dimer interface coincides with the opening of MBP. They deduced this from the spin-spin interaction between spin labels: one on MBP and the other on the transmembrane domain. In a vanadatetrapped transition state intermediate, all free MBP became tightly bound to MaIFGK2 and spin labels in both lobes were completely immobilized. In addition spin-spin interactions were lost, suggesting that MBP was in an open conformation.

Building on Austermuhle et al.'s previous work, Orelle et al. [32] discovered that both MBP and ATP are required for the closure of the nucleotide binding domain (NBD) MalK. Again, the use of SDSL and cW-EPR spectroscopy was used to study the opening and closing of the NBD. They found that after ATP hydrolysis the NBD is in a semi-open configuration, which is distinctly different from the open state. They propose that the release of inorganic phosphate $(\mathrm{Pi})$ happens concurrently with the reorientation of the transmembrane domain to an inward-facing conformation.

Grote et al. [33] used doubly spin-labelled mutants of the maltose transporter MalFGK2-E to further investig- ate the mechanism of the transport cycle mediated by transmembrane signalling. The EPR data revealed that MBP is bound throughout the transport cycle. The reciprocal communication across the membrane gives information on the effect MaIE has on ATPase activity, as they regard it to be an important mechanistic feature of receptor-coupled $A B C$ transporters.

They suggested that further characterization of the inter- domain relationships during substrate transport is needed to elucidate details of the conformational changes brought about by the SBP. They suggest that studies of spin-labelled MalE variants should be undertaken to investigate distances between positions within both the SBP and the transporter. They note that although their model is specific to the maltose transporter, similarities may be found in the BtuCD- F complex. 
Although it is often used as a model for type 1 ABC transporters, MalFGK2 has some distinct differences from the vast majority of other transporters of this kind. As such, other transporters have been studied using EPR. One such system is the histidine transporter, HisQMP2. Sippach et al. [34] used DEER (or PELDOR) spectroscopy to study the conformational changes of HisQMP2. The system works with a SBP similar to MalE, HisJ, that has a high affinity for histidine. Their results show that the distances measured resemble those of the maltose transporter throughout the cycle. In the presence of HisJ, the closed conformation of the NBDs is found. These observed conformational changes lead them to propose that there are three different conformations of NBD; open, semi-open and closed. These conformations are regulated by SBP binding.

\section{Figure 1}

A schematic view of how EPR can be used to study membrane transporters using in silico attachment of spin label rotamer libraries to the crystal structures of P-glycoprotein (Mus musculus, PDB 3G5U, left) and MsbA (Salmonella enterica, PDB 3B60, right)

Clusters in red demonstrate the use of cw-EPR mobility studies to investigate local structure and topology; pink shows the use of accessibility studies and paramagnetic quenchers to elucidate local environment; blue presents the use of pulsed EPR for distance measurements to study conformational dynamics.

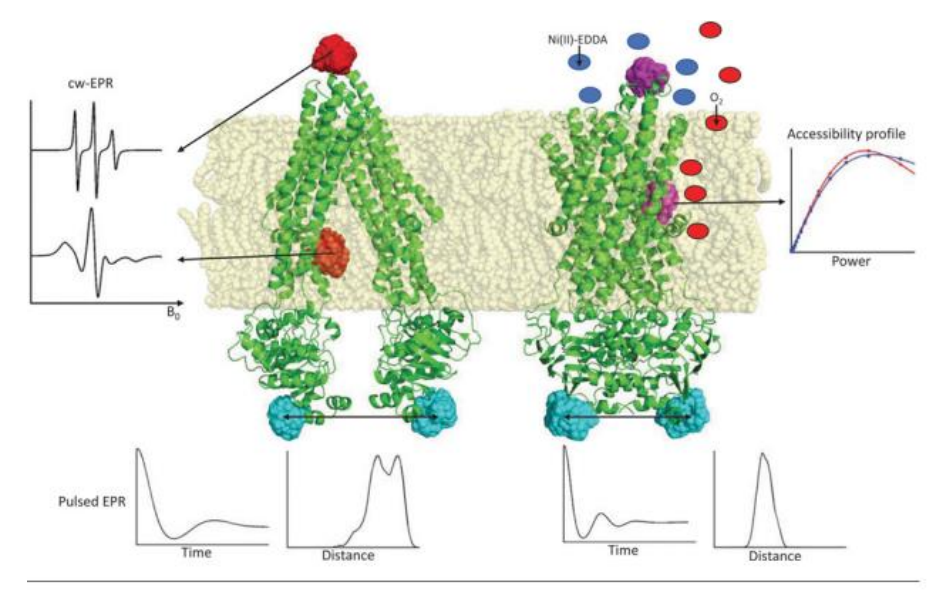

\section{Figure 2}

A comparison of different types of transmembrane transporter

(a) The multidrug transporter P-glycoprotein (Caenorhabditis elegans, PDB 4F4C), an ATP-powered pump; (b) the uniporter cyclodextrin glycosyltransferase (Bacillus

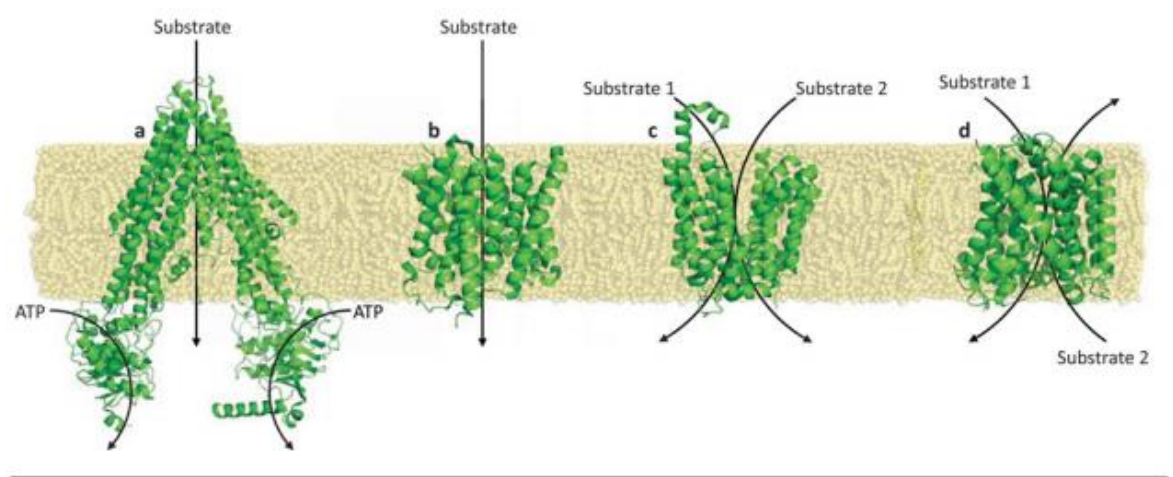




\section{Import}

Pneumococcal surface adhesin A, PsaA, plays a vital role in the virulence and colonization of the Streptococcus pneumoniae pathogen [35] and is a vital component of the Mn2 + -specific ABC-type transporter, PsaBCA permease [36]. The Mn2 + ion serves to negate the effects of oxidative stress on the protein by neutralizing reactive oxygen species (ROS) [37].

A combination of SDSL and EPR approaches were taken to investigate the protein environment. In order to determine the conformational flexibility of the PsaA protein, five PsaA variants (L56C, S58C, S266C, I125C and I236C) were labelled with the nitroxide MTSL and characterized using CW-EPR. The combination of MD simulations and CW-EPR spectra allowed for elucidation of the flexibility of the PsaA protein lobes, hypothesizing various interactions with other proteins comprising the PsaABC complex [38]. Since the intrinsic metal $(\mathrm{Mn} 2+)$ is also paramagnetic, these single variants can be used for distance determination using multi- or rather frequency ( 34 and $94 \mathrm{GHz}$ ) cw-EPR as has previous been demonstrated [39].

The MolBC type II importer transports molybdate ions in bacteria, vital for carbon and nitrogen cycling [40]. Cw- EPR spectroscopy was applied to MolBC reconstituted into proteoliposomes to elucidate the transport mechanism; MTSL was used to label the $\mathrm{S180C}$ and $\mathrm{D} 173 \mathrm{C}$ residues on gates of MolB, which are responsible for the transfer of molybdate ions through the transporter. It was deduced that in the presence of ATP the conformation of the periplasmic gate converts to an open position allowing passage of the substrate. This was seen through an increase in mobility at the D173C label position. Once ATP was hydrolysed a shift back to the original conformation was noted. The MolBC-S180C in complex with substrate-free MolA was seen to decrease spin label mobility; however, on addition of ATP, the S180C label demonstrated increased mobility [41].

PELDOR and $\mathrm{CW}$-EPR have been applied to the maltose ABC transporter MalEFGK2 [33]. MalF-P2 domain double mutants were spin labelled, along with a triple mutant with sites present in the MalK and P2 domains. For the MalF-P2 double mutant, interspin distances were determined using both CW-EPR and pulsed EPR methods, allowing for determination of rearrangements of the protein during ion transport. Furthermore, the labelled MalK domain showed communication between protein domains, and analysis of the changes of conformation in the cytoplasmic and periplasmic domains of the $A B C$ transporter highlighted the importance of communication between these two domains for transport. Shifts in spectra were evident on binding showing the dependence of ATP and MalE for conformational change of the protein [33].

The conformations undertaken by the BtuCD-F ABC transporter have also been elucidated through EPR analysis of spin-labelled mutants reconstituted in proteoliposomes. This complex is responsible for the translocation of vitamin B12 in Escftericftia coli [42,43]. Resulting spectra of BtuCD compared with BtuCD-F highlighted the differences in conformation adopted. After the addition of BtuF-B12, coupling between spin labels at residues 141 and 168 within BtuC was seen, suggesting that the distance between the two labels had decreased [44].

\section{Efflux}

The multidrug exporter MsbA shares sequence similarity with a class of $A B C$ transporters, which are linked to multidrug resistance and cancer $[45,46]$. PELDOR studies have revealed large-scale movement in opposite directions in the periplasmic and cytoplasmic parts of the transporter upon ATP hydrolysis. A $33 \mathrm{~A}^{\circ}$ change in distance was measured upon formation of the trapped postATP hydrolysis intermediate, and inhibited structural changes caused by lipopolysaccharide (LPS) binding. Results also indicate that ATP hydrolysis powers transport of LPS into an open 
cytoplasmic chamber before its translocation by alternating access, involving conformational changes of $10-20 A^{\circ}$ [47].

The Cus CFBA efflux system within E. coli is a copper- regulating system which helps maintain cellular concentra- tions [48,49]. Combinations of $\mathrm{CW}$-EPR and PELDOR have revealed the importance of residues $\mathrm{M} 36$ and $\mathrm{M} 38$ of CusB in both $\mathrm{Cu}(\mathrm{I})$ coordination to the CusBNT ( $\mathrm{N}$-terminal) domain, and interaction with CusF. Additionally, it was found that K32 is essential for interaction with CusF mutation removed the exchange interaction, suggesting differences in protein folding and separation of CusBNT monomers. It is thought that mutations of lysine residues might affect the conformational structure of CusBNT, thereby interfering with $\mathrm{Cu}(\mathrm{l})$ coordination [50].

LmrA is a multidrug $A B C$ transporter isolated from Lactococcus lactis that extrudes hydrophobic drugs from the membrane [51]. Initial EPR studies involved labelling the TMDs in order to analyse the relationship of drug recognition, transport and coupling with the hydrolysis cycle [52]. PELDOR later showed that LmrA samples far fewer conformational states upon nucleotide binding when compared with its apo state; ATP binding alone, rather than hydrolysis, is sufficient to trigger this change. It is only when the protein cycles back to its apo state that this relatively fixed conformation is lost. Results strongly suggest that alternating between two states, with distinct differences in dynamics and structure, is necessary for substrate translocation [53].

Another ABC-type efflux pump of interest is ABCB1 (also referred to as P-glycoprotein, or P-gp), which confers anticancer therapy resistance. In one study mechanistic details were elucidated from changes in the mobility and accessibility of spin-labelled transport substrate verapamil with ABCB1 reconstituted into liposomes [54]. Following this, a study used spin-labelled ATP to specifically investigate the structure and dynamics of the NBDs [55]; results of this support a two-state model of a resting open conformation with readily accessible NBDs and an ATP-trapped transition- like state where the nucleotide is buried in the protein. Doubly labelled variants of P-gp in proteoliposomes were used with PELDOR to obtain distance constraints [56]. Measurements revealed disagreement with crystal structure data [57]; this, combined with the broad distance distributions indicated high protein mobility. Shorter NBD interdomain distances were measured following ATP addition. cW-EPR accessibility experiments were performed on a range of spin- labelled variants in the resting, nucleotide-bound and post- hydrolytic states [58]. Results were rationalized using MD simulations and supported models of the protein with a central cavity involved in an alternating access mechanism.

\section{Facilitated diffusion/secondary active transport}

Secondary active transport describes the movement of substrates using the electropotential difference of a con- centration gradient across the membrane; the movement of ions along their concentration gradient (i.e. facilitated diffusion) allows the second solute to be transported against its own electrochemical gradient (i.e. active transport). The simultaneous transport of the ion and its coupled substrate can occur either together in the same direction (symport) or in opposite directions (antiport). Multidrug and toxic compound extrusion or multidrug antimicrobial extrusion (MATE) proteins, small multidrug resistance (SMR) proteins and the major facilitator superfamily (MFS) represent three of the five major classes of bacterial multidrug efflux transporters $[59,60]$; specifically, these proteins are responsible for the efflux of harmful or toxic compounds via coupled proton or sodium cation antiport. 


\section{Symport}

One of the best known groups of cotransporters is the ubiquitous sodium-solute symport superfamily (SSS, comprises 11 subfamilies), which utilize the energy harnessed from sodium motive force to drive the transport of the second solute against its concentration gradient [61].

One of these, PutP ( $\mathrm{Na}+$ /proline symporter) has been studied using a range of different EPR techniques. The earliest study exclusively used RT cW-EPR on a range of spin-labelled variants to probe label mobility and accessibility [62]. This study supported the then-recently proposed 13-helix model [63] and focused on several transmembrane domains and loops; spin labels predicted to be buried in the TMs were more restricted and less accessible than those attached to residues proposed to sit exposed on the surface with the least restricted and most accessible labels located towards the middle of the loop regions. Only 2 of the 17 variants indicated any spectral change upon substrate binding, pointing towards the possibility that the associated areas (TM II and loop 2) undergo binding-induced conformational change.

A later study used a combination of $\mathrm{CW}$-EPR and PELDOR to measure interspin distances and how they change upon substrate binding [64]. None of the double Cys variants demonstrated the dipolar interactions associated with interspin distances $<1.8 \mathrm{~nm}$; PELDOR was required to measure distances of $\sim 2-8 \mathrm{~nm}$. However, the data obtained for one pair were contradictory to the cW-EPR data, due to loop regions being flexible; the broad nature of the associated distance distributions supports this explanation. The L4/L7 distance was measured to be $4.8 \mathrm{~nm}$; this agrees with the previous model, indicating that these loops are on opposite sides of the membrane. Subsequent addition of sodium and proline caused minimal changes.

Another study focused on the structure of the backbone of TM IX, using PELDOR to measure 16 double Cys variants [65]. This TM domain had recently been suggested to be involved in the translocation pathway [66]. The results of the measurements determined that this transmembrane domain was kinked; the bend was suggested to act as a hinge, sealing the inward-facing cavity upon substrate binding and opening for substrate release into the cell.

A more recent study specifically targeted extracellular loop 4 (EL4), proposed to act as a gate to ligand binding sites $[10,67]$; the study used cw-EPR to investigate mobility, polarity and accessibility profiles, and PELDOR to measure interspin distances of PutP reconstituted into liposomes. The results of these studies show that EL4 consists of two $\alpha$ - helices connected by a loop region (similar to LeuT and VSGLT). One of the residues within EL4 is demonstrated to have hydrophobic contact with the CTM1 (ten of the TMs of the 13-helix model are 'core', or CTMs), sealing the extracellular gate upon substrate binding.

Another member of the SSS is Mhp1, a Na+ /benzyl-hydantoin symporter, believed to operate via the alternating access model $[68,69]$. A single EPR study on this system has been published, using PELDOR to determine interspin distances to investigate the movement of transmembrane domains under different ligand conditions [70]. The results of the study indicate that sodium binding does not induce a conformational change; instead it is the second substrate (in the presence of sodium) that shifts the conformational equilibrium. This is contrary to previously published crystal structures and suggests that LeuT-fold proteins may have at least two different coupling mechanisms.

The small hydrophobic amino acid transporter LeuT is a bacterial homologue of the neurotransmitter/sodium symporter (NSS) family, one of the subgroups that make up the SSS. It is responsible for the cotransport of leucine and sodium cations and is a homodimer [71] although this is disputed. Only a single EPR group has studied this protein, using mobility and accessibility 
experiments alongside measurements of distance constraints [e.g. 72]. These studies reported results that suggested that the crystal structures at the time represented inhibited conformations.

BetP, a Na+/glycine betaine symporter, is one of the most studied osmo-regulated uptake systems; it is another member of the LeuT-fold group of proteins, physiologically existing as an asymmetric homotrimer [73,74]. An EPR study using $\mathrm{cw}$ - and pulsed EPR methods [75] resulted in determination that the C-terminal domain weakly interacts either with corresponding domains in adjacent BetP monomers, or with the lipid bilayer (the latter agreeing with a proposed functional model) [76]. It was also suggested that the packing within the trimer determined from experimental distance constraints differs significantly from that seen in the crystal structures.

The galactose permease GalP system is involved in monosaccharide transport using chemiosmosis. It is a member of the MFS; it has a 12 transmembrane $\alpha$-helix fold with both the N-terminus and Cterminus located in the cytoplasm [77]. Crystallography of the protein reconstituted into liposomes shows that it exists functionally in trimers [78]. Conventional cw-EPR (i.e. label mobility studies) and saturation transfer EPR techniques showed that the labelling site was oriented into a densely packed interhelical region and that the packing of the helices is less tight than the proposed models [79]. Prior to the publication of any crystal data, EPR was used to investigate the stoichiometry and selectivity of the lipids around the protein in a membrane environment [80].

Another member of the MFS is LacY, a $\mathrm{H}+/ \beta$-galactoside cotransporter; the crystal structure was first solved in 2003 [81], with various structures published since; it is purported to function via the alternating access model [82]. Distance measurements performed using PELDOR appear to support this assertion, with ligand-induced conformational changes resulting in opposite movements of the cytoplasmic and periplasmic ends of the transmembrane bundles [83].

GltPh is found within chemical synapses and couples aspartate transport to the symport of three sodium ions within synapses. PELDOR measurements on twotrimerization-domain mutants taken for both the apoprotein, and the protein in the presence of coupling ions and substrate indicated that the trimerization-domain forms the stable core. Measurements performed on mutants of transporting domains showed sampling of multiple conformations in all states to similar extents; this is consistent with large- scale movement during the transport cycle. Conformations favoured in the membrane environment are different from those favoured in detergent micelles [84]. A simultaneous study by Georgieva et al. [85] demonstrated that the domain motions involved in the outward-to-inward transition occur both in detergent and in membranes, and energies of the outward-facing and inward-facing states were similar both in the presence and absence of substrate.

\section{Antiport}

Among the most studied and best characterized antiport systems is NhaA of $\mathrm{E}$. coli, a $\mathrm{Na}+/ \mathrm{H}+$ cotransporter thought to regulate $\mathrm{pH}$ and use proton motive force to expel sodium $[86,87]$. $\mathrm{cW}$ - and pulsed EPR techniques were used to confirm the assertion that this protein can exist as a dimer [88]. Further experiments suggested that the dimerization equilibrium is moderately $\mathrm{pH}$-dependent. Building on this, NhaA was reconstituted into liposomes in conjunction with PELDOR to determine the physiological dimer structure [89]. Two points of contact within the homodimer were found, contradicting preceding high-resolution crystal and cryo-EM data (though it is stated that further investigation with varying $\mathrm{pH}$ is required to confirm or refute these claims). $\mathrm{NHE1}$, or $\mathrm{Na}+/ \mathrm{H}+$ exchanger isoform 1, is one of nine mammalian isoforms similar in structure and function to the bacterial NhaA protein; studies of this protein have been based on the structural model of NhaA, with EPR being used to map and measure distance constraints to support this [90]. OxIT, a member 
of the MFS, is responsible for the exchange of oxalate for formate in Oxalobacter formigenes [91]. Much of the work done on this system has been modelled on other members of the MFS, many of which are mentioned in this review (e.g. LacY, GalP etc.). PELDOR was used to measure long-range distances and it was found that OxIT adopts the inward-open and outward-open states in the presence of substrate [92]; this is contradictory to previous homology- modelled predictions, which suggested the conformation in which the cytoplasmic side is sealed and periplasmic side is open would not be significantly populated.

Pho84 is an MFS phosphate/H+ antiporter of Saccftaro-myces cerevisiae. The crystal structure of the protein has not been solved, but has been modelled on other MFS members; a cw-EPR label mobility study has been used to investigate the accuracy of a homology-modelled structure using GItP [93]. Results were promising, suggesting the model was accurate, though further studies would be required to confirm this as not many sites on the protein were sampled.

LmrP is an MFS multidrug transporter from L. lactis that couples proton translocation to the extrusion of cytotoxic molecules [94]. Initial EPR studies undertaken gave evidence of distinct structural changes upon ligand binding orprotonation of specific residues; - most profound was the rearrangement of helix XIII upon substrate binding, strongly indicating that the C-and N-terminal interface plays an important role in controlled drug access [95]. Subsequent cW-EPR and PELDOR studies suggest alternation between outward-open and outward-closed conformations, caused by protonation of specific residues, allowing a transmembrane protonation relay. A model was proposed involving the initiation of transport via substrate binding and opening of the extracellular side, after which specific residue protonation causes substrate release on the extracellular side. This also causes a number of conformational changes leading to proton transfer and release to the intracellular side [96].

NorM of Vibrio paraftaemolyticus was the first multidrug and toxic compound extrusion (MATE) protein to be classified and is one of the best-studied so far; several conserved acidic residues in membrane-embedded regions have been identified that take part in Na+-coupled transport [97] but the mechanism of substrate binding could not be elucidated from crystal structures.

MATE proteins were first categorized for bacteria but have also been found in mammalian and plant cells and are believed to be universally present in all living organisms [59,98]. It has been demonstrated that MATE proteins play an important role in antimicrobial resistance of bacteria, making them an attractive potential target for novel antimicrobial and anticancer drugs [99], whereas in plants they contribute to homoeostasis by secretion of waste products and detoxification of metals, and in mammalian cells it is likely that they mediate final excretion of toxic organic cations [98].

Many MATE proteins are known to be $\mathrm{Na}+$ /drug antiporters although some transporters have been discovered that use protons instead of sodium cations, like PmpM of Pseudomonas aeruginosa or hMATE1 in human liver, kidney and skeletal muscle cells [59].

MATE proteins show a wide range of substrate specificity among which fluoroquinolones like norfloxacin are sub- strates for almost all of the transporters. Each transporter can pump out several of a growing list of identified compounds with very different chemical structures, from cationic dyes and intercalators (e.g. ethidium bromide or doxorubicin) to aminoglycosides (e.g. kanamycin and streptomycin) or $\beta$ - lactam antibiotics (e.g. ampicillin) [59].

The structure of MATE family proteins shows 12-13 $\alpha$-helical transmembrane segments (TMS) [100]. Steed et al. published an EPR study on Vc-NorM, using the spin-labelled substrate homologue 
Ruboxyl as paramagnetic probe to gather information on location and mechanism of substrate binding and expected translocation of substrate in $\mathrm{Na}+$ presence. Quantitative results for substrate binding (both in the presence and absence of competitors) and $\mathrm{Na}$--dependence were gained from $\mathrm{CW}$-EPR lineshape experiments. Multiple modes of substrate binding were detected from the data, with one high affinity binding site and non-specific binding in case of substrate excess.

DEER experiments were used to locate the high-affinity binding site. Vc-NorM was spin-labelled at six periplasmic sites and one cytoplasmic site in order to identify the substrate position in crystal structure scaffolds of Vc-NorM [101] and Ng-NorM [102]. Results point to one membrane-embedded high-affinity substrate-binding site at TMS 7, close to the loop at TMS 7/8 that probably shifts upon substrate/ion binding. CW-EPR lineshapes also showed that addition of known substrates as competitors for Ruboxyl binding led to reduction in Ruboxyl binding to the high-affinity binding site to approximately $50 \%$.

Highly interesting was the discovery that $\mathrm{Na}+$ concentration, varied from 0 to $100 \mathrm{mM}$, did not have any effect on substrate binding nor did it trigger substrate translocation. The lack of Na+ dependence for Ruboxyl binding supports the theory that MATE transporters (or at least NorM) do not operate according to the classical mechanism of antiport but via a transport cycle comprising multiple equilibrium states that depend on a $\mathrm{Na}+$ gradient rather than $\mathrm{Na}+$ concentration.

SMR proteins are specific for a vast variety of substrates, including quaternary ammonium compounds (QAC), other lipophilic cations and a multitude of detergents, antiseptics, cationic dyes and antibiotics [103-105].

Main substrates of EmrE are tertiary and quaternary bulky aromatic cations and other positively charged hydrophobic compounds. Substrates bind at Glu-14 embedded in TMS 1 and the extrusion mechanism using the pmf is well characterized, although it is not yet fully understood on a structural basis. EPR and SDSL were used to further elucidate the structure and its conformations involved in the transport cycle.

The oligomerization state was investigated by Koteiche et al. (2003) using cw-EPR and SDSL, concluding in agreement with other studies that it forms a homodimer [106,107]. The question of topology of the dimers is still discussed as arguments for both parallel and antiparallel topology can be found [108-110]. Both cw-EPR [106] and pulsed EPR [111] of spin-labelled EmrE pointed towards a parallel topology which would also be the conformation with least bias within the membrane. More recent cw-EPR results though [112] support antiparallel topology. In this study, spin labels were introduced along the axes of the TM helices to gain more insight in topology and conformational changes upon substrate binding. Accessibility assays with $\mathrm{O} 2$ and NiEDDA (Ni(II)ethylenediamine-N,Nr-diacetic acid) with and without TPP+ as substrate were performed. Both NiEDDA and $\mathrm{O} 2$ function as paramagnetic relaxants and their collision frequency with spin label, resulting in characteristic changes in EPR spectra, allows conclusions on the environment of the particular spin label. NiEDDA is only soluble in aqueous phases whereas $\mathrm{O} 2$ enters only the membranes and thus, membrane boundaries and lipid- facing or water-exposed residues can be identified. EPR results showed, that a putative symmetric interchange from apo to substrate-bound intermediate could not be verified by the data. Instead, a permeation pathway of the substrate through the asymmetric dimer was suggested. Both for further elucidation of substrate transport by EmrE and for the still unresolved question of topology, Amadi et al. suggest long range distance measurements. 
EmrD is a member of the MFS. MFS transporters are ubiquitous; in bacteria they are mainly used for nutrition uptake and extrusion of harmful compounds. They consist of 12 TM helices, have two pseudosymmetrical halves and likely transport substrates via alternating accessibility of a central cavity.

EmrD from E. coli is the only structurally characterized multidrug/ $\mathrm{H}+$ antiporter (DHA) of the MFS so far $[1,113,114]$. The crystal structure revealed a doubly occluded conformation with unexpected features and an EPR study [114] followed to assess the structure in a more native-like environment in unilamellar liposomes. In this study, $76 \mathrm{EmrD}$ mutants with spin labels introduced along their TM helical axes were investigated using cw-EPR. Information gathered from EPR lineshape and from accessibility assays with $\mathrm{O} 2$ and NiEDDA were mostly consistent with the crystal structure, although major deviations were found for the orientation of TMS 5 and the topology of TMS 10 and 11.

Furthermore, EPR results showed $\mathrm{pH}$-dependent con- formational changes that led to opening of a cytoplasmic cleft at TMS 2-4 in the N-terminal half of EmrD, a region containing the MFS signature GxxxD(R/K)xG and conserved amino acid residues Asp-68 and Asp-123 that could play a crucial role in $\mathrm{H}+$ transport, and alongside mediated movement of TMS 6 that reduced accessibility at the periplasmic side. Thus, Steed et al. could confirm a pH- dependent conformational switch in EmrD as expected for a multidrug/ $\mathrm{H+}$-antiporter. Structural data on substrate binding and transport by EmrD in vitro and in cell-based assays the authors questioning the suitability of EmrD as a model for other MFS DAH transporters.

\section{Conclusions}

Knowledge of protein structures at atomic resolution is essential to understand function. Although crystallography remains the mainstream method to obtain structural inform- ation, crystal structures of dynamic membrane transport proteins are difficult to derive, and often crystallography only provide static snapshots. Indeed, very few membrane proteins have been crystallized in more than one conformation. Knowledge of such structural and conformational changes is a key to understand how membrane transporters translocate substrates across the membrane EPR spectroscopy can, in principle, provide such complex dynamic information over a large range of distances, allowing for measurement of distances, environment and protein dynamics. Taken together with the structural information of protein snapshots from crystals, EPR has the power to enhance our understanding of the complex functional dynamics at play in important macromolecular protein complexes such as membrane transporters.

Late Professor Steve Baldwin was at the forefront of embracing such new techniques and recognizing the power of new and complementary approaches to resolving structure/function/dynamics relationships in complex molecular machines. With this review we hope to demonstrate that, just as Steve recognized, EPR is well on its way to becoming fully integrated into the structural biologist's arsenal of tools.

\section{Acknowledgements}

We acknowledge the COST Action (CM1306) 'Understanding Movement and Mechanism in Molecular Machines' for continued financial support.

\section{Funding}

This work was supported by the Wellcome Trust [grant numbers 094392/B/10/Z (to F.M.), xxx (to J.H.) and xxx (to I.H.)]; the Royal Society Wolfson Research Merit Award [grant number xxx (to F.M.)]; 
the Biochemical Society for a summer studentship bursary, COST [CM1306] [grant number xxx (to A.S.M.)]; the UEA [grant numbers $x x x$ (to A.S.M.) and $x x x$ (to A.F.)].

\section{References}

1. Quistgaard, E.M., Lo“ w, C., Guettou, F. and Nordlund, P (2016) Understanding transport by the major facilitator superfamily (MFS): structures pave the way. Nat. Rev. Mol. Cell Biol. 17, 123-132

2. Comellas, G. and Rienstra, C.M. (2013) Protein structure determination by magic-angle spinning solid-state NMR, and insights into the formation, structure, and stability of amyloid fibrils. Annu. Rev. Biophys. 42, 515-536

3. Krishnamurthy, H. and Gouaux, E. (2012) X-ray structures of LeuT in substrate-free outwardopen and apo inward-open states. Nature 481, 469-474

4. Husada, F., Gouridis, G., Vietrov, R., Schuuman-Wolters, G.K., Ploetz, E., de Boer, M., Poolman, B. and Cordes, T. (2015) Watching conformational dynamics of $A B C$ transporters with single molecule tools. Biochem. Soc. Trans. 43, 1041-1047

5. Do" rr, J.M., Koorengevel, M.C., Scha" fer, M., Prokofyev, A.V., Scheidelaar, S., van der Cruijsen, E.A.W., Dafforn, T.R., Baldus, M. and Killian, J.A. (2014) Detergent-free isolation, characterization, and functional reconstitution of a tetrameric $\mathrm{K}+$ channel: the power of native nanodiscs. Proc. Natl. Acad. Sci. U.S.A. 111, 18607-18612

6. Milov, A.D., Salikhov, K.M. and Shirov, M.D. (1981) Application of the double resonance method to electron spin echo in a study of the spatial distribution of paramagnetic centres in solids. Sov. Phys. Solid State 23, 565-569

7. Martin, R.E., Pannier, M., Diederich, F., Gramlich, V., Hubrich, M. and Speiss, H.W. (1998) Determination of end-to-end distances in a series of TEMPO diradicals of up to 2-8 nm length with a new four-pulse double electron-electron resonance experiment. Angew. Chem. Int. Ed. 37, 2834-2837

8. Borbat, P.P., McHaourab, H. and Freed, J.H. (2002) Protein structure determination using long-distance constraints from double-quantum coherence ESR: study of T4 lysozyme. J. Am. Chem. Soc. 124, 5304-5314

9. Prisner, T.F., Rohrer, M. and MacMillan, F. (2001) Pulsed EPR spectroscopy: biological applications. Annu. Rev. Phys. Chem. 52, 279-313

10. Klare, J.P. and Steinhoff, H.-J. (2015) Chapter eleven - Spin labeling studies of transmembrane signaling and transport: applications to phototaxis, $A B C$ transporters and symporters. Meth. Enzymol. 564, 315-347

11. Jeschke, G. (2012) DEER distance measurements on proteins. Annu. Rev. Phys. Chem. 63, 419-446

12. Borbat, P.P. and Freed, J.H. (2013) Pulse dipolar electron spin resonance: distance measurements. In In Structure and Bonding (Timmel, C.R. and Harmer, J., eds), vol. 152, pp. 1-82, Springer, Heidelberg

13. (a) Yang, Z., Bridges, M., Lerch, M.T., Altenbach, C. and Hubbell, W.L. (2015) Saturation recovery EPR and nitroxide spin labeling for exploring structure and dynamics in proteins. Methods Enzymol. 564, 3-27 (b) Lerch, M.T., Yang, Z., Altenbach, C. and Hubbell, W.L. (2015) High- pressure EPR and site-directed spin labeling for mapping molecular flexibility in proteins. Methods Enzymol. 564, 29-57 (c) Altenbach, C., Lo' pez, C.J., Hideg, K. and Hubbell, W.L. (2015) Continuous-wave electron paramagnetic resonance spectroscopy. Methods Enzymol. 564, 59-100 
14. Polyhach, Y., Bordignon, E., Tschaggelar, R., Gandra, S., Godt, A. and Jeschke, G. (2012) High sensitivity and versatility of the DEER experiment on nitroxide radical pairs at Q-band frequencies. Phys. Chem. Chem. Phys. 14, 10762-10773

15. Spindler, P.E., Glaser, S.J., Skinner, T.E. and Prisner, T.F. (2013) Broadband inversion PELDOR spectroscopy with partially adiabatic shaped pulses. Angew. Chem. Int. Ed. 52, 3425-3429

16. Hirst, S.J., Alexander, N., McHaourab, H.S. and Meiler, J. (2011) RosettaEPR: an integrated tool for protein structure determination from sparse EPR data. J. Struct. Biol. 173, 506-514

17. Jeschke, G., Chechik, V., Ionita, P., Godt, A., Zimmermann, H., Banham, J., Timmel, C.R., Hilger, D. and Jung, H. (2006) DeerAnalysis2006: a comprehensive software package for analysing pulsed ELDOR data. Appl. Magn. Reson. 30, 473-498

18. Polyhach, Y., Bodignon, E. and Jeschke, G. (2011) Rotamer libraries of spin labelled cysteines for protein studies. Phys. Chem. Chem. Phys. 13, 2356-2366

19. Ward, R., Bowman, A., Sozudogru, E., El-Mkami, H., Owen-Hughes, T. and Norman, D.G. (2010) EPR distance measurements in deuterated proteins. J. Magn. Reson. 207, 164-167

20. van Wonderen, J.H., Kostrz, D.N., Dennison, C. and Macmillan, F. (2013) Refined distances between paramagnetic centers of a multi-copper nitrite reductase determined by pulsed EPR (iDEER) spectroscopy. Angew. Chem. Int. Ed. Engl. 52, 1990-1993

21. Abe, C., Klose, D., Dietrich, F., Ziegler, W.H., Polyhach, Y., Jeschke, G. and Steinhoff, H.-J. (2012) Orientation selective DEER measurements on vinculin tail at $X$-band frequencies reveal spin label orientations. J. Magn. Reson. 216, 53-61

22. Reginsson, G.W., Hunter, R.I., Cruickshank, P.A., Bolton, D.R., Sigurdsson, S.T., Smith, G.M. and Schiemann, O. (2012) W-band PELDOR with $1 \mathrm{~kW}$ microwave power: molecular geometry, flexibility and exchange coupling. J. Magn. Reson. 216, 175-182

23. Tkach, I., Pornsuwan, S., Hobartner, C., Wachowius, F., Sigurdsson, S.T., Baranova, T.Y., Diederichsen, U., Sicoli, G. and Bennati, M. (2013) Orientation selection in distance measurements between nitroxide spin labels at $94 \mathrm{GHz}$ EPR with variable dual frequency irradiation. Phys. Chem. Chem. Phys. 15, 3433-3437

24. Yang, Z., Liu, Y., Borbat, P., Zweier, J.L., Freed, J.H. and Hubbell, W.L. (2012) Pulsed ESR dipolar spectroscopy for distance measurements in immobilized spin labeled proteins in liquid solution. J. Am. Chem. Soc. 134, 9950-9952

25. Krstic' , I., Ha“" nsel, R., Romainczyk, O., Engels, J.W., Do“" tsch, V. and Prisner, T.F. (2011) Long-range distance measurements on nucleic acids in cells by pulsed EPR spectroscopy. Angew. Chem. Int. Ed. 50, 5070-5074

26. Azarkh, M., Okle, O., Singh, V., Seemann, I.T., Hartig, J.S., Dietrich, D.R. and Drescher, M. (2011) Long-range distance determination in a DNA model system inside Xenopus laevis oocytes by in-cell spin-label EPR. Chem. Bio. Chem. 12, 1992-1995

27. Wilkinson, A.J. (2002) In In ABC Proteins: From Bacteria to Man (Holland, B., Kuchler, K., Cole, S.P. and Higgins, C., eds), Elsevier Science and Technology Books, London28

28. Gonin, S., Arnoux, P., Pierru, B., Lavergne, J., Alonso, B., Sabaty, M. and Pignol, D. (2007) Crystal structures of an extracytoplasmic solute receptor from a TRAP transporter in its open and closed forms reveal a helix-swapped dimer requiring a cation for alpha-keto acid binding. BMC Struct. Biol. 7, 11

29. Neiditch, M.B., Federle, M.J., Pompeani, A.J., Kelly, R.C., Swem, D.L., Jeffrey, P.D., Bassler, B.L. and Hughson, F.M. (2006) Ligand-induced asymmetry in histidine sensor kinase complex regulates quorum sensing. Cell 126, 1095-1108

30. Hall, J.A., Ganesan, A.K., Chen, J. and Nikaido, H. (1997) Two modes of ligand binding in maltose-binding protein of Escherichia coli. J. Biol. Chem. 272, 17615-17622 
31. Austermuhle, M.I., Hall, J.A., Klug, C.S. and Davidson, A.L. (2004) Maltose-binding protein is open in the catalytic transition state for ATP hydrolysis during maltose transport. J. Biol. Chem. 279, 28243-28250

32. Orelle, C., Ayvaz, T., Everly, R.M., Klug, C.S. and Davidson, A.L. (2008) Both maltose-binding protein and ATP are required for nucleotide-binding domain closure in the intact maltose ABC transporter. Proc. Natl. Acad. Sci. U.S.A. 105, 12837-12842

33. Grote, M., Polyhach, Y., Jeschke, G., Steinhoff, H.J., Schneider, E. and Bordignon, E. (2009) Transmembrane signaling in the maltose ABC transporter MalFGK2-E. J. Biol. Chem. 284, 17521-17526

34. Sippach, M., Weidlich, D., Klose, D., Abe' , C., Klare, J., Schneider, E. and Steinhoff, H.J. (2014) Conformational changes of the histidine ATP-binding cassette transporter studied by double electron-electron resonance spectroscopy. Biochim. Biophys. Acta 1838, 1760-1768

35. Clatworthy, A.E., Pierson, E. and Hung, D.T. (2007) Targeting virulence: a new paradigm for antimicrobial therapy. Nat. Chem. Biol. 3, 541-548

36. Bajaj, M., Mamidyala, S., Zeugg, J., Begg, S., Ween, M., Luo, Z., Huang, J.X., McEwan, A.G., Kobe, B., Paton, J.C. et al. (2015) Discovery of novel pneumococcal surface antigen A (PsaA) inhibitors using a fragment-based drug design approach. ACS Chem. Biol. 10, 1511-1520

37. McDevitt, C., Ogunniyi, A., Valkov, E., Lawrence, M., Kobe, B., McEwan, A. and Paton, J.C. (2011) A molecular mechanism for bacterial susceptibility to zinc. PLoS Pathog 7, e1002357

38. Deplazes, E., Begg, S.L., Wonderen, J.H., Campbell, R., Kobe, B., Paton, J.C., MacMillan, F., McDevitt, C.A. and O'Mara, M.L. (2015) Characterizing the conformational dynamics of metal-free PsaA using molecular dynamics simulations and electron paramagnetic resonance spectroscopy. Biophys. Chem. 207, 51-60

39. Ka" ss, H., MacMillan, F., Ludwig, B. and Prisner, T.F. (2000) Investigation of the Mn Binding Site in cytochrome c oxidase by high-frequency EPR. J. Phys. Chem. B 104, 5362-5371

40. Hille, R. (1996) The mononuclear molybdenum enzymes. Chem. Rev. 96, 2757-2816

41. Rice, A.J., Harrison, A., Alvarez, F.J.D., Davidson, A.L. and Pinkett, H.W. (2014) Small substrate transport and mechanism of a molybdate ATP binding cassette transporter in a lipid environment. J. Biol. Chem. 289, 15005-15013

42. Reynolds, P.R., Mottur, G.P. and Bradbeer, C. (1980) Transport of vitamin B12 in Escherichia coli. Some observations on the roles of the gene products of btuc and tonb. J. Biol. Chem. 255,4313

43. Veaux, L.C., Clevenson, D.S., Bradbeer, C. and Kadner, R.J. (1986) Identification of the btuCED polypeptides and evidence for their role in vitamin B12 transport in Escherichia coli. J. Bacteriol 167, 920

44. Hvorup, R.N., Goetze, B.A., Niederer, M., Hollenstein, K., Perozo, E. and Locher, K.P. (2007) Asymmetry in the structure of the $A B C$ transporter-binding protein complex BtuCD-BtuF. Science 317, 1387-1390

45. Gottesman, M.M., Fojo, T. and Bates, S.E. (2002) Multidrug resistance in cancer: role of ATPdependent transporters. Nat. Rev. Cancer 2, 48-59

46. van Veen, H.W., Callaghan, R., Soceneantu, L., Sardini, A., Konings, W.N. and Higgins, C.F. (1998) A bacterial antibiotic-resistance gene that complements the human multidrugresistance P-glycoprotein gene. Nature 391, 291-295

47. Borbat, P.P., Surendhran, K., Bortolus, M., Zou, P., Freed, J.H. and McHaourab, H.S. (2007) Conformational motion of the ABC transporter MsbA induced by ATP hydrolysis. PLoS Biol. $10,2211-2219$

48. Delmar, J.A., Su, C.C. and Yu, E.W. (2013) Structural mechanisms of heavy-metal extrusion by the Cus efflux system. BioMetals 26, 593-607 
49. Franke, S., Grass, G., Rensing, C. and Nies, D.H. (2003) Molecular analysis of the coppertransporting efflux system CusCFBA of Escherichia coli. J. Bacteriol 185, 3804-38012

50. Meir, A., Natan, A., Moskovitz, Y. and Ruthstein, S. (2015) EPR spectroscopy identifies Met and Lys residues that are essential for the interaction between the CusB N-terminal domain and Metallochaperone CusF. Metallomics 7, 1163-1172

51. van Veen, H.W., Venema, K., Bolhuis, H., Oussenko, I., Kok, J., Poolman, B., Dreissen, A. and Konings, W.N. (1996) Multidrug resistance mediated by a bacterial homolog of the human multidrug transporter MRD1. Proc. Natl. Acad. Sci. U.S.A. 93, 10668-10672

52. Hellmich, U.A., Lyubenova, S., Moenkemeyer, L., Kaltenborn, E., van Veen, H.W., Prisner, T. and Glaubitz, C. (2009) Investigation Of the multidrug ABC-transporter LmrA By multinuclear MAS-NMR and EPR. Biophys. J. 96, 594a

53. Hellmich, U.A., Lyubenova, S., Kaltenborn, E., Doshi, R., van Veen, H.W., Prisner, T.F. and Glaubitz, C. (2012) Probing the ATP hydrolysis cycle of the ABC multidrug transporter LmrA by pulsed EPR spectroscopy. J. Am. Chem. Soc. 134, 5857-5862

54. Omote, H. and Al-Shawi, M.K. (2002) A novel electron paramagnetic resonance approach to determine the mechanism of drug transport by P-glycoprotein. J. Biol. Chem. 277, 4568845694

55. Delannoy, S., Urbatsch, I.L., Tomboline, G., Senior, A.E. and Vogel, P.D. (2005) Nucleotide binding to the multidrug resistance P-glycoprotein as studied by ESR spectroscopy. Biochemistry 44, 14010-14019

56. Wen, P.-C., Verhalen, B., Wilkens, S., Mchaourab, H.S. and Tajkhorshid, E. (2013) On the origin of large flexibility of P-glycoprotein in the inward-facing state. J. Biol. Chem. 288, 19211-19220

57. Aller, S.G., Yu, J., Ward, A., Weng, Y., Chittaboina, S., Zhuo, R., Harrel, P.M., Trinh, Y.T., Zhang, Q., Urbatsch, I.L. and Chang, G. (2009) Structure of P-glycoprotein reveals a molecular basis for poly-specific drug binding. Science 323, 1718-1722

58. van Wonderen, J.H., McMahon, R., O'Mara, M.L., McDevitt, C.A., Thomson, A.J., Kerr, I.D., MacMillan, F. and Callaghan, R. (2014) The central cavity of $A B C B 1$ undergoes alternating access during ATP hydrolysis. FEBS J. 281, 2190-2201

59. Kuroda, T. and Tsuchiya, T. (2009) Multidrug efflux transporters in the MATE family. Biochim. Biophys. Acta 1794, 763-768

60. Bay, D.C., Rommens, K.L. and Turner, R.J. (2008) Small multidrug resistance proteins: a multidrug transporter family that continues to grow. Biochim. Biophys. Acta 1778, 18141838

61. Reizer, J., Reizer, A. and Saier, M.H. (1994) A functional superfamily of sodium/solute symporters. Biochim. Biophys. Acta 1197, 133-166

62. Wegener, C., Tebbe, S., Steinhoff, H.-J. and Jung, H. (2000) Spin labeling analysis of structure and dynamics of the $\mathrm{Na}+$ /proline transporter of Escherichia coli. Biochemistry 39, 48314837

63. Jung, H., Ru“ benhagen, R., Tebbe, S., Liefker, K., Tholema, N., Quick, M. and Schmid, R. (1998)

64. Jeschke, G., Wegener, C., Nietschke, M., Jung, H. and Steinhoff, H.-J. (2004) Interresidual distance determination by four-pulse double electron-electron resonance in an integral membrane protein: the $\mathrm{Na}+$ /proline transporter PutP of Escherichia coli. Biophys. J. 86, 2551-2557

65. Hilger, D., Polyhach, Y., Jung, H. and Jeschke, G. (2009) Backbone structure of transmembrane domain IX of the $\mathrm{Na}+$ /proline transporter PutP of Escherichia coli. Biophys.

J. 96, 217-225 
66. Hilger, D., Bo“ hm, M., Hackmann, A. and Jung, H. (2008) Role of Ser340 and Thr341 in transmembrane domain IX of the $\mathrm{Na}+$ /proline transporter PutP of Escherichia coli in ligand binding and transport. J. Biol. Chem. 283, 4921-4929

67. Raba, M., Dunkel, S., Hilger, D., Lipiszko, K., Polyhach, Y., Jeshke, G., Bracher, S., Klare, J.P., Quick, M., Jung, H. and Steinhoff, H.-J. (2014) Extracellular loop 4 of the proline transporter PutP controls the periplasmic entrance to ligand binding sites. Structure 22, 769-780

68. Weyand, S., Shimamura, T., Yajima, S., Suzuki, S., Mirza, O., Krusong, K., Carpenter, E.P., Rutherford, N.G., Hadden, J.M., O'Reilly, J. et al. (2008) Structure and molecular mechanism of a nucleobase-cation-symport-1 family transporter. Science 322, 709-713

69. Shimamura, T., Weyand, S., Beckstein, O., Rutherford, N.G., Hadden, J.M., Sharples, D., Sansom, M.S.P., Iwata, S., Henderson, P.J.F. and Cameron, A.D. (2010) Molecular basis of alternating access membrane transport by the sodium-hydantoin transporter Mhp1. Science $328,470-473$

70. Kazmier, K., Sharma, S., Islam, S.M., Roux, B. and Mchaourab, H.S. (2014) Conformational cycle and ion-coupling mechanism of the $\mathrm{Na}+$ /hydantoin transporter Mhp1. Proc. Natl. Acad. Sci. U.S.A. 111, 14752-14757

71. Krishnamurthy, H. and Gouaux, E. (2012) X-ray structures of LeuT in substrate-free outwardopen and apo inward-open states. Nature 481, 469-474

72. Claxton, D.P., Quick, M., Shi, L., Delmondes de Carvalho, F., Weinstein, H., Javitch, J.A. and Mchaourab, H.S. (2010) lon/substrate-dependent conformational dynamics of a bacterial homolog of neurotransmitter: sodium symporters. Nat. Struct. Mol. Biol. 17, 822-829

73. Ressl, S., Terwisscha van Scheltinga, A.C., Vonrhein, C., Ott, V. and Ziegler, C. (2009) Molecular basis of transport and regulation in the $\mathrm{Na}+/$ betaine symporter BetP. Nature 458, 47-52

74. Tsai, C.J., Khafizov, K., Hakulinen, J., Forrest, L.R., Kra“ mer, R., Ku“ hlbrandt, W. and Ziegler, C. (2011) Structural asymmetry in a trimeric $\mathrm{Na}+$ /betaine symporter, BetP, from Corynebacterium glutamicum. J. Mol. Biol. 407, 368-381

75. Nicklisch, S.C.T., Wunnicke, D., Borovykh, I.V., Morbach, S., Klare, J.P., Steinhoff, H.-J. and Kra" mer, R. (2012) Conformational changes of the betaine transporter BetP from Corynebacterium glutamicum studied by pulse EPR spectroscopy. Biochim. Biophys. Acta 1818, 359-366

76. Ott, V., Koch, J., Spa" te, K., Morbach, S. and Kra“ mer, R. (2008) Regulatory properties and interaction of the $\mathrm{C}$ - and $\mathrm{N}$-terminal domains of BetP, an osmoregulated betaine transporter from Corynebacterium glutamicum. Biochemistry 47, 12208-12218

77. Saier, M.H., Beatty, J.T., Goffeau, A., Harley, K.T., Heijne, W.H., Huang, S.-C., Jack, D.L., Ja“ hn, P.S., Lew, K., Liu, J. et al. (1999) The major facilitator superfamily. J. Mol. Microbiol. Biotechnol. 1, 257-279

78. Zheng, H., Taraska, J., Merz, A.J. and Gonen, T. (2010) The prototypical H + /galactose symporter GalP assembles into functional trimers. J. Mol. Biol. 396, 593-601

79. Marsh, D. and Henderson, P.J.F. (2001) Specific spin labelling of the sugar-H + symporter, GalP, in cell membranes of Escherichia coli: site mobility and overall rotational diffusion of the protein. Biochim. Biophys. Acta 1510, 464-473

80. Hubert, A., Henderson, P.J.F. and Marsh, D. (2003) Lipid-protein interactions in Escherichia coli membranes overexpressing the sugar-H + symporter, GalP EPR of spin-labelled lipids. Biochim. Biophys. Acta 1611, 243-248

81. Abramson, J., Smirnova, I., Kasho, V., Verner, G., Kaback, H.R. and Iwata, S. (2003) Structure and mechanism of the lactose permease of Escherichia coli. Science 301, 610-615 
82. Smirnova, I., Kasho, V. and Kaback, H.R. (2011) Lactose permease and the alternating access mechanism. Biochemistry 50, 9684-9693

83. Smirnova, I., Kasho, V., Choe, J.-Y., Altenbach, C., Hubbell, W.L. and Kaback, H.R. (2007) Sugar binding induces an outward facing conformation of LacY. Proc. Natl. Acad. Sci. U.S.A. 104, 16504-16509

84. Ha" nelt, I., Wunnicke, D., Bordignon, E., Steinhoff, H.-J. and Slotboom, D.J. (2013) Conformational heterogeneity of the aspartate transporter GltPh. Nat. Struct. Mol. Biol. 20, 210-214

85. Georgieva, E.R., Borbat, P.P., Ginter, C., Freed, J.H. and Boudker, O. (2013) Conformational ensemble of the sodium-coupled aspartate transporter. Nat. Struct. Mol. Biol. 20, 215-222

86. Padan, E., Venturi, M., Gerchman, Y. and Dover, N. (2001) Na + /H+ antiporters. Biochim. Biophys. Acta 1505, 144-157

87. Hunte, C., Screpanti, E., Venturi, M., Rimon, A., Padan, E. and Michel, H. (2005) Structure of a $\mathrm{Na}+/ \mathrm{H}+$ antiporter and insights into mechanism of action and regulation by $\mathrm{pH}$. Nature 435 , 1197-1202

88. Hilger, D., Jung, H., Padan, E., Wegener, C., Vogel, K.-P., Steinhoff, H.-J. and Jeschke, G. (2005) Assessing oligomerization of membrane proteins by four-pulse DEER: pH-dependent dimerization of $\mathrm{NhaA} \mathrm{Na}+/ \mathrm{H}+$ antiporter of E-coli. Biophys. J. 89, 1328-1338

89. Hilger, D., Polyhach, Y., Padan, E., Jung, H. and Jeschke, G. (2007) High-resolution structure of a $\mathrm{Na}+/ \mathrm{H}+$ antiporter dimer obtained by pulsed electron paramagnetic resonance distance measurements. Biophys. J. 93, 3675-3683

90. Nygaard, E.B., Lagerstedt, J.O., Bjerre, G., Shi, B., Budamagunta, M., Poulsen, K.A., Meinild, S., Rigor, R.R., Voss, J.C., Cala, P.M. and Pedersen, S.F. (2011) Structural modeling and electron paramagnetic resonance spectroscopy of the human $\mathrm{Na}+/ \mathrm{H}+$ exchanger isoform 1 , NHE1. J. Biol. Chem. 286, 634-648

91. Anantharam, V., Allison, M.J. and Maloney, P.C. (1989) Oxalate:formate exchange. The basis for energy coupling in Oxalobacter. J. Biol. Chem. 264, 7244-7250

92. Iyalomhe, O., Herrick, D.Z., Cafiso, D.S. and Maloney, P.C. (2014) Closure of the cytoplasmic gate formed by TM5 and TM11 during transport in the oxalate/formate exchanger from Oxalobacter formigenes. Biochemistry 53, 7735-7744

93. Lagerstedt, J.O., Voss, J.C., Wieslander, A. and Persson, B.L. (2004) Structural modeling of dual-affinity purified Pho84 phosphate transporter. FEBS Lett. 578, 262-268

94. Putman, M., van Veen, H.W., Degener, J.E. and Konings, W.N. (2001) The Lactococcal secondary multidrug transporter LmrP confers resistance to lincosamides, macrolides, streptogramins and tetracyclines. Microbiology 147, 2873-2880

95. 95 Masureel, M., Smriti, S., Martens, C., Zou, P., Ruysschaert, J.-M., Mchaourab, H.S. and Govaerts, C. (2012) Studying the conformational cycle of the secondary multidrug transporter LmrP by EPR spectroscopy. Biophys. J. 102, 660A

96. Masureel, M., Martens, C., Stein, R.A., Mishra, S., Ruysschaert, J.-M., Mchaourab, H.S. and Govaerts, C. (2014) Protonation drives the conformational switch in the multidrug transporter LmrP. Nat. Chem. Biol. 10, 148-155

97. Otsuka, M., Yasuda, M., Morita, Y., Otsuka, C., Tsuchiya, T., Omote, H. and Moriyama, Y. (2005) Identification of essential amino acid residues of the NorM Na + /multidrug antiporter in Vibrio parahaemolyticus. J. Bacteriol 187, 1552-1558

98. Moriyama, Y., Hiasa, M., Matsumoto, T. and Omote, H. (2008) Multidrug and toxic compound extrusion (MATE)-type proteins as anchor transporters for the excretion of metabolic waste products and xenobiotics. Xenobiotica 38, 1107-1118

99. Van Veen, H.W. (2010) Last of the multidrug transporters. Nature467, 926-927 
100. Steed, P.R., Stein, R.A., Mishra, S., Goodman, M.C. and Mchaourab, H.S. (2013) Na + substrate coupling in the multidrug antiporter NorM probed with a spin-labeled substrate. Biochemistry 52, 5790-5799

101. He, X., Szewczyk, P., Evin, M., Hong, W.-X., Zhang, Q. and Chang, G. (2010) Structure of a cation-bound multidrug and toxic compound extrusion transporter. Nature 467, 991-994

102. Lu, M., Symersky, J., Radchenko, M., Koide, A., Guo, Y., Nie, R. and Koide, S. (2012) Structures of a $\mathrm{Na}+$-coupled, substrate-bound MATE multidrug transporter. Proc. Natl. Acad. Sci. U.S.A. 110, 2099-2104

103. Littlejohn, T.G., DiBerardino, D., Messerotti, L.J., Spiers, S.J. and Skurray, R.A. (1991) Structure and evolution of a family of genes encoding antiseptic and disinfectant resistance in Staphylococcus aureus. Gene 101, 59-66

104. Paulsen, I.T., Skurray, R.A., Tam, R., Saier, Jr, M.H., Turner, R.J., Weiner, J.H., Goldberg, E.B. and Grinius, L.L. (1996) The SMR family: a novel family of multidrug efflux proteins involved with the efflux of lipophilic drugs. Mol. Microbiol. 19, 1167-1175

105. Jack, D.L., Storms, M.L., Tchieu, J.H., Paulsen, I.T. and Saier, Jr, M.H. (2000) A broadspecificity multidrug efflux pump requiring a pair of homologous SMR-type proteins. J. Bacteriol. 182, 2311-2313

106. Koteiche, H.A., Reeves, M.D. and McHaourab, H.S. (2003) Structure of the substrate binding pocket of the multidrug transporter EmrE: site-directed spin labeling of transmembrane segment 1 . Biochemistry 42, 6099-6105

107. Schuldiner, S. (2009) EmrE, a model for studying evolution and mechanism of ion-coupled transporters. Biochim. Biophys. Acta 1794, 748-762

108. Korkhov, V.M. and Tate, C.G. (2009) An emerging consensus for the structure of EmrE. Acta Crystallogr. D. Biol. Crystallogr. 65, 186-192

109. Steiner-Mordoch, S., Solomon, M.D., Rotem, D., Gold, A., Yechieli, M., Adam, Y. and Schuldiner, S. (2008) Parallel topology of genetically fused EmrE homodimers. EMBO J 27, 17-26

110. Hellmich, U.A. and Glaubnitz, C. (2009) NMR and EPR studies of membrane transporters. Biol. Chem. 390, 815-834

111. Mchaourab, H.S., Mishra, S., Koteiche, H.A. and Amadi, S.H. (2008) Role of sequence bias in the topology of the multidrug transporter EmrE. Biochemistry 47, 7980-7982

112. Amadi, S.T., Koteiche, H.A., Mishra, S. and McHaourab, H.S. (2010) Structure, dynamics, and substrate-induced conformational changes of the multidrug transporter EmrE in liposomes.

J. Biol. Chem. 285, 26710-26718

113. Yan, N. (2015) Structural biology of the major facilitator superfamily transporters. Ann. Rev. Biophys. 44, 257-283

114. Steed, P.R., Zou, P., Trone, K.E. and Mchaourab, H.S. (2013) Structure and pH-Induced structural rearrangements of the putative multidrug efflux pump EmrD in liposomes probed by site-directed spin labeling. Biochemistry 52, 7964-7974 